Western University

Scholarship@Western

Bone and Joint Institute

$9-1-2019$

\title{
Performance of QCT-Derived scapula finite element models in predicting local displacements using digital volume correlation
}

\author{
Jonathan Kusins \\ Western University \\ Nikolas Knowles \\ Western University \\ Melissa Ryan \\ The University of Sheffield \\ Enrico Dall'Ara \\ The University of Sheffield \\ Louis Ferreira \\ Western University
}

Follow this and additional works at: https://ir.lib.uwo.ca/boneandjointpub

Part of the Medicine and Health Sciences Commons

Citation of this paper:

Kusins, Jonathan; Knowles, Nikolas; Ryan, Melissa; Dall'Ara, Enrico; and Ferreira, Louis, "Performance of QCT-Derived scapula finite element models in predicting local displacements using digital volume correlation" (2019). Bone and Joint Institute. 442.

https://ir.lib.uwo.ca/boneandjointpub/442 


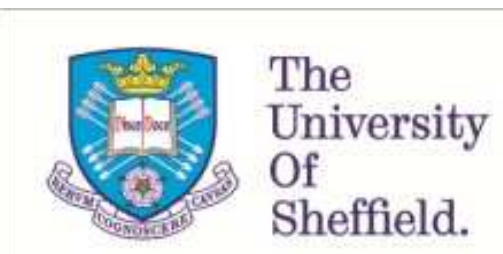

This is a repository copy of Performance of QCT-Derived scapula finite element models in predicting local displacements using digital volume correlation.

White Rose Research Online URL for this paper:

http://eprints.whiterose.ac.uk/147233/

Version: Accepted Version

\section{Article:}

Kusins, J., Knowles, N., Ryan, M. et al. (2 more authors) (2019) Performance of QCT-Derived scapula finite element models in predicting local displacements using digital volume correlation. Journal of the Mechanical Behavior of Biomedical Materials, 97. pp. 339-345. ISSN 1751-6161

https://doi.org/10.1016/j.jmbbm.2019.05.021

Article available under the terms of the CC-BY-NC-ND licence

(https://creativecommons.org/licenses/by-nc-nd/4.0/).

\section{Reuse}

This article is distributed under the terms of the Creative Commons Attribution-NonCommercial-NoDerivs (CC BY-NC-ND) licence. This licence only allows you to download this work and share it with others as long as you credit the authors, but you can't change the article in any way or use it commercially. More information and the full terms of the licence here: https://creativecommons.org/licenses/

\section{Takedown}

If you consider content in White Rose Research Online to be in breach of UK law, please notify us by emailing eprints@whiterose.ac.uk including the URL of the record and the reason for the withdrawal request. 


\title{
Performance of QCT-Derived Scapula Finite Element Models in
}

Keywords: Subject-specific finite element analysis; digital volume correlation; shoulder FEM;

${ }^{1}$ Department of Mechanical and Materials Engineering Western University London, Canada

${ }^{2}$ Roth|McFarlane Hand and Upper Limb Centre

St. Joseph's Health Care London, Canada

\section{${ }^{3}$ Department of Oncology and Metabolism}

University of Sheffield Sheffield, United Kingdom

${ }^{4}$ Insigneo Institute for In Silico Medicine

University of Sheffield

Sheffield, United Kingdom

\author{
*Correspondence Address: \\ Louis M. Ferreira, $\mathrm{PhD}$ \\ Roth|McFarlane Hand and Upper Limb Centre, \\ Surgical Mechatronics Laboratory, St. Josephs Health Care, \\ 268 Grosvenor St. London, ON, Canada. \\ Tel: + 15196466000 X. 61351 \\ E-mail address: Louis.Ferreira@sjhc.london.on.ca
}

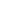


Subject-specific finite element models (FEMs) of the shoulder complex are commonly used to predict differences in internal load distribution due to injury, treatment or disease.

36 However, these models rely on various underlying assumptions, and although experimental

37 validation is warranted, it is difficult to obtain and often not performed. The goal of the current 38 study was to quantify the accuracy of local displacements predicted by subject-specific QCT-based 39 FEMs of the scapula, compared to experimental measurements obtained by combining digital 40 volume correlation (DVC) and mechanical loading of cadaveric specimens within a microCT 41 scanner.

Four cadaveric specimens were loaded within a microCT scanner using a custom-designed 43 six degree-of-freedom hexapod robot augmented with carbon fiber struts for radiolucency. 44 BoneDVC software was used to quantify full-field experimental displacements between pre- and 45 post-loaded scans. Corresponding scapula QCT-FEMs were generated and three types of boundary 46 conditions (BC) (idealized-displacement, idealized-force, and DVC-derived) were simulated for 47 each specimen.

DVC-derived BCs resulted in the closest match to the experimental results for all 49 specimens (best agreement: slope ranging from $0.87-1.09$; highest correlation: $\mathrm{r}^{2}$ ranging from 50 0.79-1.00). In addition, a two orders of magnitude decrease was observed in root-mean-square 51 error when using QCT-FEMs with simulated DVC-derived BCs compared to idealized52 displacement and idealized-force BCs. 


\section{Introduction}

56 Subject-specific finite element models (FEMs) of the shoulder complex provide the capability to

57 predict the internal load distribution of the joint. Although quantitative computed tomography

58 (QCT)-derived FEMs have been accepted as an established research tool to further understand the mechanics of the shoulder (Allred et al., 2016; Terrier et al., 2005; Zheng et al., 2017), the accuracy

60 of predictions generated by these models is to some extent unknown. Currently, experimental 61 validation of QCT-FEMs of the shoulder is limited to localized predictions of strain on the cortical

62 shell (Dahan et al., 2016; Gupta et al., 2004); while the accuracy of internal predictions within the 63 trabecular bone has yet to be explored.

To observe the internal load distribution within human bone, recent experimental protocols have combined mechanical loading with simultaneous time-lapsed volumetric imaging of bone specimens undergoing deformation (Nazarian and Müller, 2004). Digital volume correlation

67 (DVC) techniques have been introduced to quantify full-field localized displacement 68 measurements between pre- and post-loaded volumetric images (Grassi and Isaksson, 2015; Roberts et al., 2014). In addition, previous experimental studies that have combined volumetric 70 imaging with DVC analysis techniques have shown great promise to elucidate internal fracture

71 mechanisms by quantifying progressive strain and damage evolution within composite materials

72 (Croom et al., 2016, 2019, 2017). To acquire volumetric images of a deformed specimen, a CT73 compatible loading device is required. Current CT-compatible joint loading devices are based on 74 screw-type mechanisms and are restricted by the degrees-of-freedom (dof) of load they can apply 75 (Du et al., 2015; Jackman et al., 2016; Martelli and Perilli, 2018; Palanca et al., 2016; Sukjamsri

76 et al., 2015; Zhou et al., 2018). However, to properly replicate physiological joint loads at the 77 shoulder complex, a 6-dof loading mechanism would be desirable. Regardless, current 
experimental protocols developed with DVC techniques have shown tremendous promise by quantifying internal localized deformations within trabecular bone that otherwise cannot be captured experimentally (Bay et al., 1999; Chen et al., 2017; Gillard et al., 2014; Liu and Morgan, 2007).

DVC has been applied at the micro (e.g. trabecular bone cores) and joint level (e.g. vertebra) to validate $\mu \mathrm{CT}$ - and QCT-FEMs respectively (Chen et al., 2017; Costa et al., 2017; Hussein et al., 2018; Jackman et al., 2016; Mao et al., 2019, p.; Zauel et al., 2005). At the micro level, the accuracy of the predictions generated by $\mu$ CT-FEMs was found to be sensitive to the boundary condition (BC) modelled (Chen et al., 2017). Specifically, when using BCs derived directly from local DVC measurements, any inherent experimental limitations (e.g. specimen fixation rigidity or structural stiffness of the loading mechanism) were assumed to be eliminated and thus excellent agreement (slope $(\mathrm{m}) \approx 1$, coefficient of determination $\left(\mathrm{r}^{2}\right) \approx 1$, y-intercept $(\mathrm{b})$ $\approx 0$ ) was achieved (Chen et al., 2017; Costa et al., 2017; Oliviero et al., 2018). However, when extrapolating these techniques to QCT-FEMs at the joint level, similar success has not been reported. Previous studies performed by Jackman et. al. and Hussein et. al. within the vertebra found improvements in performance of QCT-FEMs when using BCs derived from DVC measurements obtained at the yield point; but only moderate agreement between the experimental local displacements and FEM predictions were observed (Hussein et al., 2018; Jackman et al., 2016). A similar validation study has yet to be performed to quantify the performance of shoulder QCT-FEMs in predicting local experimental displacement measurements obtained through DVC. Hence, the primary objective of the current study was to quantify the accuracy of local displacements predicted by subject-specific shoulder QCT-FEMs compared to experimental measurements obtained through mechanical loading and simultaneous volumetric imaging of 
101 cadaveric scapular specimens. A secondary objective was the design and fabrication of a CT102 compatible 6-dof loading apparatus capable of applying articular loads within a microCT scanner.

103

104

105

106

107

108

109

110

\section{Materials and Methods}

\subsection{Development of a CT-Compatible 6-DOF Loading Apparatus}

A custom 6-dof hexapod parallel robot was designed to apply external loads to a scapula. The robot consisted of a base, loading platform and six prismatic actuators that connected the base and platform (Figure 1) in a configuration consistent with a Stewart platform design. Although variations exist, the Stewart platform design commonly uses six linear actuators attached in pairs by universal joints to a movable platform and fixed base. Each prismatic actuator is composed of a lead screw mechanism driven by independent servo-motors. Although the robot consisted of only linear actuators, its hexapod configuration transforms linear displacements into complete 6-dof motions that include all possible translations and rotations, within its range of motion. In addition, the Stewart platform design is noted to have a high load carrying capacity within a small working envelope and has been used in previous applications involving 6-dof biomechanical testing applications (Boyin Ding et al., 2011; Lawless et al., 2014; Walker and Dickey, 2007).

The hexapod robot was augmented with radiolucent carbon fiber extensions to provide CTcompatibility with a Nikon XT H 225ST cone-beam microCT scanner. Custom fixtures were fabricated to allow for loads to be applied to the glenoid of a cadaveric scapula within a microCT scanner. A hemispherical platen (diameter equal to $48 \mathrm{~mm}$ ) was fabricated from acetal plastic and attached to the loading platform via an acrylic extension rod. A 6-dof load cell (Mini 45, ATI Industrial Automation, NC, USA) was instrumented to the loading platform to provide real-time 
122 force feedback during the experimental loading protocol. Overall, the current system can apply 1.5

$123 \mathrm{kN}$ of compression and weighs $9.6 \mathrm{~kg}$.

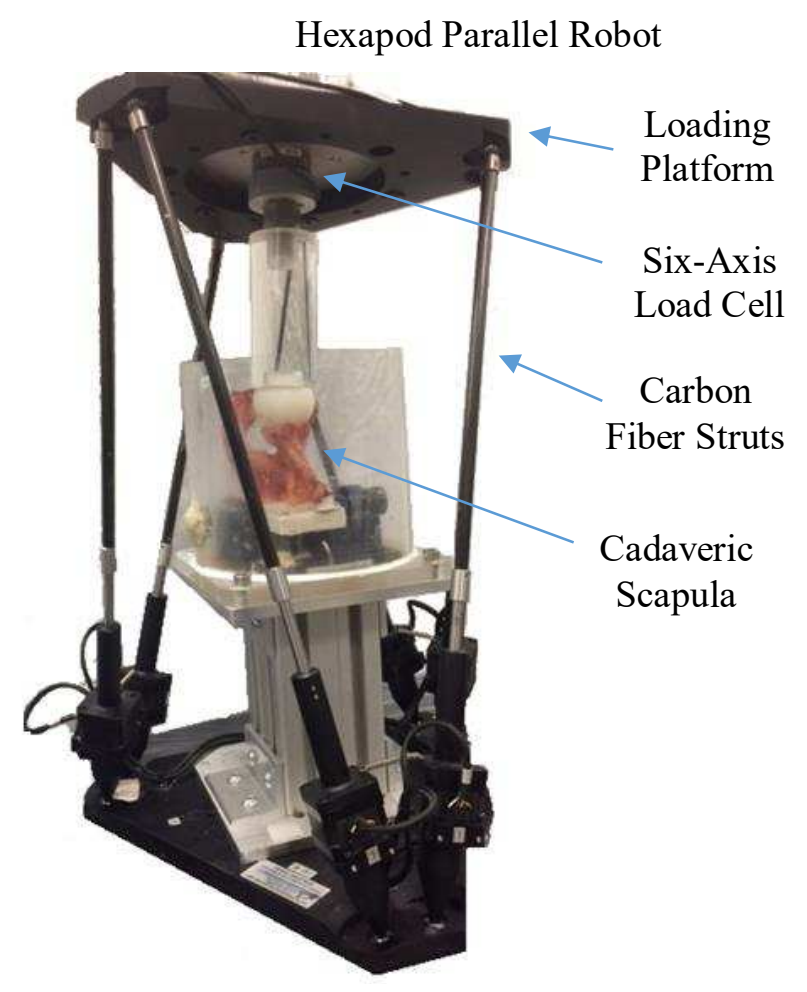

Figure 1: A custom-designed six degree-of-freedom hexapod robot was used to experimentally load cadaveric scapulae within a cone-beam microCT scanner. The use of carbon fiber struts provided radiolucent sections to reduce any imaging artifacts that may occur with use of the hexapod robot.

\subsection{Specimen Preparation and Experimental Loading}

126 Four cadaveric shoulders (two male and two female) were tested (Table 1). Prior to experimental

127 loading, the specimens with soft tissue intact were imaged within a clinical QCT-scanner (GE

128 Discovery CT750 HD, Milwaukee, WI, USA) at clinical scan settings (pixel size: $0.625 \mathrm{~mm}$ to

$1290.668 \mathrm{~mm}$, slice thickness: $0.625 \mathrm{~mm}, 120 \mathrm{kVp}, 200 \mathrm{~mA}$, BONEPLUS). A dipotassium phosphate

130 (K2HPO4) calibration phantom (QCT Pro, Mindways Software Inc., Austin, TX, USA) was 
132 at clinical resolution were used for development of continuum-level QCT-derived finite element 133 models described further in Section 2.4. Following the scanning protocol, each scapula was 134 denuded of all soft tissue. The glenoid articular surface was resurfaced using a clinical shoulder 135 reaming tool to provide a consistent uniform surface for experimental loading. The scapula was 136 then cut on a medial plane (sectioned approximately $55 \mathrm{~mm}$ from the articular surface) and 137 cemented in polymethyl methacrylate (PMMA) for fixation in the hexapod robot. A custom jig 138 was used to orient the axis at which the glenoid was resurfaced perpendicular to the loading 139 platform of the hexapod robot.

140 Table 1: Age, sex, and QCT density ( \pm 1 SD) of cadaveric scapulae specimens.

\begin{tabular}{l|ccc}
\hline & Sex & Age & QCT Density $\left(\mathrm{g}_{\text {K2 } \mathrm{HPO}} / \mathrm{cm}^{3}\right)$ \\
\hline Specimen 1 & Male & 80 & $0.333 \pm 0.256$ \\
Specimen 2 & Male & 73 & $0.245 \pm 0.198$ \\
Specimen 3 & Female & 62 & $0.376 \pm 0.240$ \\
Specimen 4 & Female & 52 & $0.377 \pm 0.253$ \\
\hline
\end{tabular}
scanner (Nikon XT H 225ST). Each specimen was wrapped with tissue-soaked phosphate-buffered 143 saline solution to ensure hydration throughout the scanning protocol. The hexapod robot, 144 previously described, was used to apply external loads. Consistent for each specimen, an initial 145 stabilizing load (10 N, settling time of 20 minutes to allow for specimen relaxation) was applied 146 and a pre-loaded microCT scan was acquired $(33.5 \mu \mathrm{m}$ isotropic voxel size, $95 \mathrm{kVp}, 64 \mu \mathrm{A}, 3141$ 147 projections, 55 minute scan time, $1000 \mathrm{~ms}$ exposure). Two post-loaded scans of the specimen were 148 then obtained with identical settings as the pre-loaded scan. The first load consisted of a target 149 compressive load of $500 \mathrm{~N}$ with a settling time of 20 minutes. The second load consisted of a target $150500 \mathrm{~N}$ load applied off-axis, $5^{\circ}$ posterior for each specimen and $5^{\circ}$ inferior for specimens 1,2 , and 1514 with respect to the robot's frame. An inferior off-axis angle was not applied to specimen 3 due 
152 to interference between the acrylic extension rod and the acromion of the specimen. Both loading

153 cases were performed directly within the microCT scanner, without repositioning the loading

154 apparatus between scans. Load cell measurements were acquired via a NI-USB 6210 data

155 acquisition unit (National Instruments Corporation, Austin, Texas) obtained after the 20 minutes

156 settling time but prior to the microCT scan. The resulting field of view (FOV) for each scan was a

157 cube with edge lengths of $65 \mathrm{~mm}$, which captured the glenoid vault (approximately $25 \mathrm{~mm}$ medial

158 from the articular surface) and the loading platen in the pre- and post-loaded states.

\subsection{Digital Volume Correlation}

160 Local experimental displacement measurements between the pre- and post-loaded images were 161 obtained using DVC algorithms (Figure 2). To prepare the images for DVC, a specimen-specific 162 threshold was applied (Mimics v.20.0, Materialise, Leuven, BE) to segment and isolate the glenoid 163 vault from other objects captured within the microCT scans (e.g. loading platen). Values outside 164 the selected threshold were assigned a constant grey level value similar to bone marrow (equivalent 165 to 85 in 8-bit greyscale). The images were then cropped and converted to 8-bit greyscale (ImageJ, 166 NIH) (Schneider et al., 2012).

A previously established and validated deformable image registration toolkit, BoneDVC, was used to quantify the full-field experimental displacement field between the pre- and post-

169 loaded images (Dall'Ara et al., 2017, 2014). BoneDVC is a global based DVC approach that 170 computes full-field local displacement vectors between two sets of volumetric images using cross 171 correlation techniques (Dall'Ara et al., 2014). Furthermore, BoneDVC has previously been used 172 to validate $\mu$ FEMs for various osseous structures (Chen et al., 2017; Costa et al., 2017; Oliviero et 173 al., 2018). To compute the precision of the local displacement measurements quantified by 
174 BoneDVC, a standard procedure of comparing two pre-loaded scans with various nodal spacing 175 was performed (Dall'Ara et al., 2017, 2014). Based on these results, a nodal spacing of 30 voxels, 176 (approximately $1 \mathrm{~mm}$ ), was decided as an optimal tradeoff between spatial resolution and precision 177 (error along $\mathrm{x}, \mathrm{y}$, and $\mathrm{z}$ direction lower than $2.5 \mu \mathrm{m}$ ).

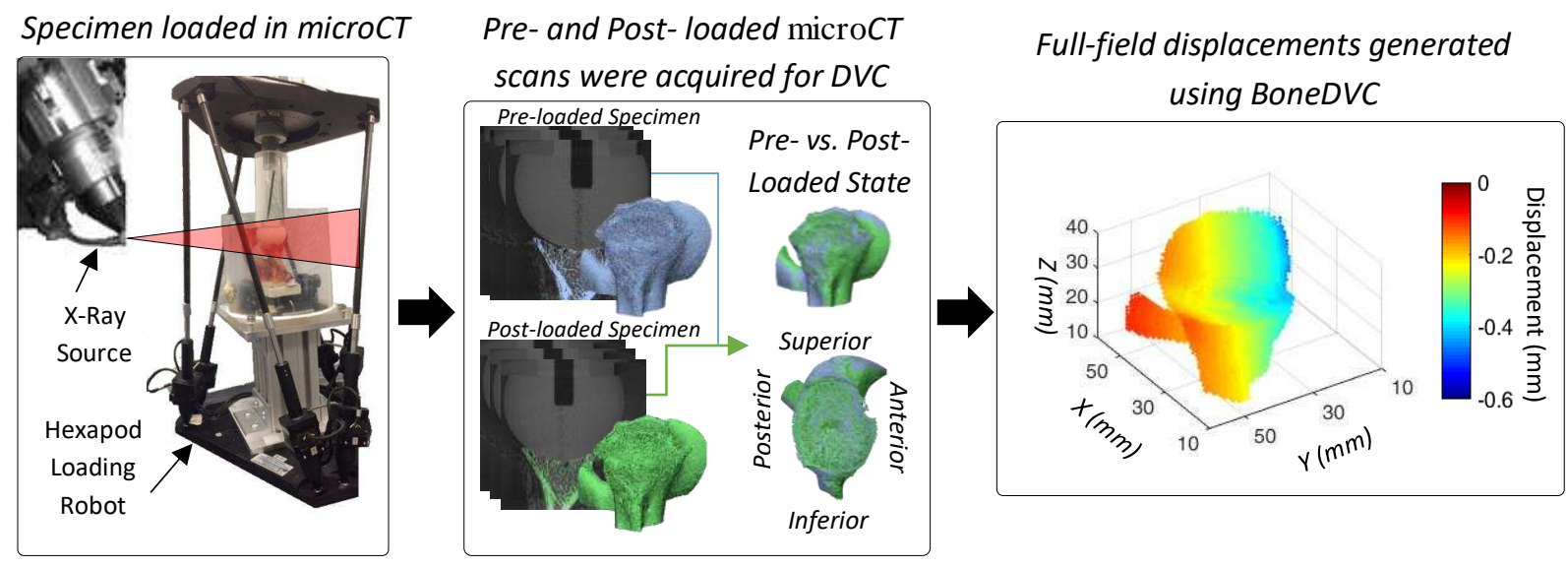

Figure 2: Four scapula specimens were scanned within a microCT at a resolution of $33.5 \mu \mathrm{m}$ in pre- and postloaded states. Full-field local displacements (sub-volume size $\approx 1 \mathrm{~mm}$ ) between the pre- and post-loaded scans were obtained using BoneDVC.

\subsection{Computational In-Silico Modelling}

Specimen-specific QCT-FEMs were generated for each scapula to simulate the experimental loading set-up. The geometry of each scapula was extracted from the corresponding QCT scan acquired prior to experimental loading. To identify the medial border at which the experimental scapulae were cut, laser surface scans (Artec Spider, Artec 3D, Luxembourg) were acquired for each prepared specimen. From these, STL models were generated and registered to the QCT models. Anything below the PMMA surface was removed by Boolean subtraction. In addition, any elements within the volume of the reamed articular surface were removed to match the

187 prepared cadaveric scapulae. A surface triangular mesh was generated (3-matic v.12.0, 188 Materialise, Leuven, BE) with a target edge length of $1 \mathrm{~mm}$ (Burkhart et al., 2013). The surface 
mesh was then converted to a quadratic tetrahedral mesh using ABAQUS (v.6.14, Simulia,

190

Providence, RI). Linear elastic isotropic material properties were applied to the volumetric mesh (Mimics v.20.0, Materialise, Leuven, BE) based on the local density measure using Eq.1 and Eq. 2 (Rice et al., 1988; Schaffler and Burr, 1988).

$$
\begin{array}{lc}
\rho_{\text {app }}<1.54 \mathrm{~g} / \mathrm{cm}^{3} & E_{\text {trab }}=60+900 * \rho_{a p p}{ }^{2} \\
\rho_{\text {app }} \geq 1.54 \mathrm{~g} / \mathrm{cm}^{3} & E_{\text {cort }}=90 * \rho_{a p p}{ }^{7.4}
\end{array}
$$

Where $\mathrm{E}_{\text {trab }}$ is Young's modulus of trabecular bone [MPa], $\mathrm{E}_{\text {cort }}$ is Young's modulus of cortical bone $[\mathrm{MPa}]$, and $\rho_{\text {app }}$ is apparent density.

To register the QCT-FEMs to the coordinate system of the microCT, an iterative closest points algorithm (3-matic Research 11.0, Materialise, Leuven, BE) was performed aligning the outer geometry of the QCT-derived scapula to the corresponding microCT-derived scapula (Knowles et al., 2019).

Three separate boundary conditions (BCs) were modelled (ABAQUS v.6.14, Simulia, Providence, RI) to investigate their effect on the accuracy of the QCT-FEMs. The first two simulations consisted of idealized BCs (idealized-displacement BC and idealized-force BC). For both idealized BCs, a deformable virtual loading platen, meshed with hexahedral elements $(\mathrm{E}=$ $3100 \mathrm{MPa}, v=0.35)$, was constructed and general contact between the virtual platen and scapula was modelled (coefficient of friction $=0.2$ ). The medial border of the scapula was assumed to be fixed and either a force (idealized-force BC) or displacement (idealized-displacement BC) was applied to the virtual loading platen (Figure 3). For the idealized-force BC, the experimental force measured immediately prior to the microCT scan was prescribed to the top nodes of the virtual loading platen. For the idealized-displacement BC, a displacement was prescribed to the top nodes of virtual loading platen forcing the platen to the post-loaded experimental position. The 
experimental post-loaded position of the loading platen was quantified by segmenting out the experimental platen in the corresponding raw post-loaded microCT image.

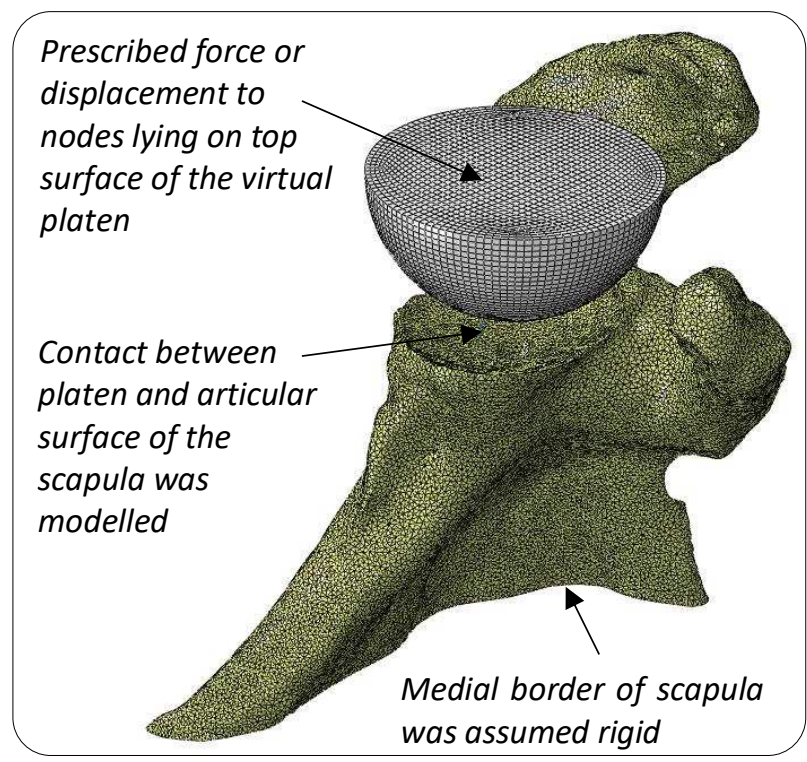

Figure 3: Specimen-specific QCT-FEMs with idealized BCs were modelled. Idealized BCs assumed the medial border of the scapula was fixed and either a displacement (idealized-displacement) or force (idealized-force) was applied to the virtual loading platen.

In addition, DVC-derived BCs were modelled (Chen et al., 2017; Costa et al., 2017;

214 Jackman et al., 2016; Zauel et al., 2005) (Figure 4). First, to generate the DVC-derived BCs, each 215 specimen was further cropped medially due to the limited FOV of the microCT scans. 216 Subsequently, local displacements were prescribed to each node lying on the articular surface of 217 the glenoid and the medial surface of the cropped scapula. A custom Matlab code (v.R2017a, 218 Mathworks, Natick, MA) applied tri-linear interpolation to local displacements provided by the 219 DVC measurements onto the corresponding QCT-FEM nodes. 


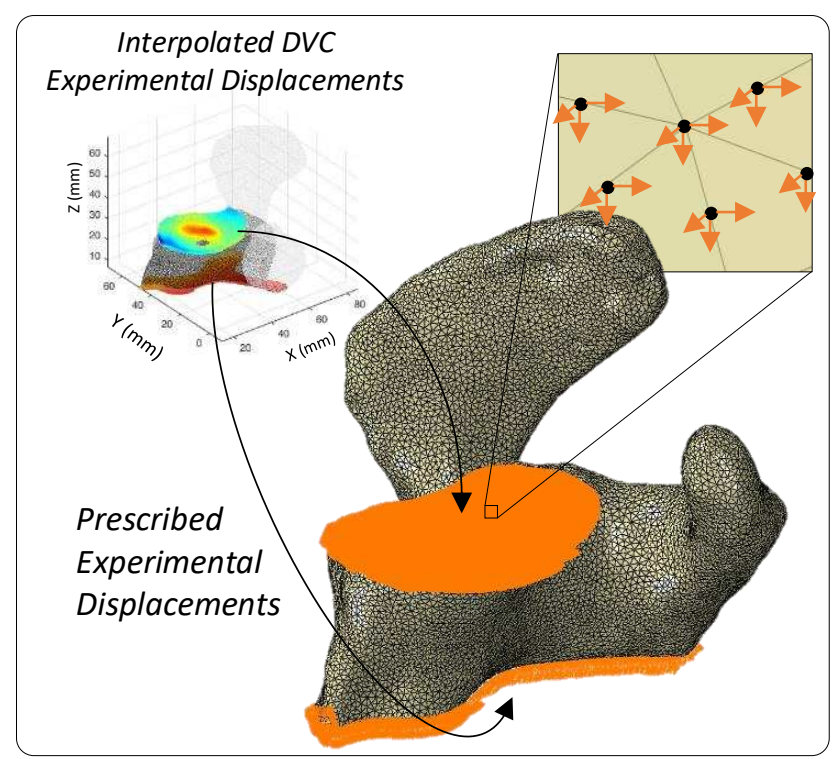

Figure 4: DVC-derived BCs were modelled by assigning local experimental displacements obtained by DVC directly to the articular and medial surface of the QCT-FEM scapula.

\subsection{Statistical Analysis}

222 To quantify the performance of the scapula QCT-FEMs, local displacement predictions were 223 compared to DVC experimental measurements using linear regression. To pair the outcome 224 measures, local DVC displacements were paired with averaged QCT-FEM predicted 225 displacements, region averaged within a $1 \mathrm{~mm}$ cubic voxel, equivalent to the DVC nodal spacing 226 and dependent on the nodal location of the DVC measurement (Hussein et al., 2018; Jackman et 227 al., 2016). Furthermore, to exclude any measurements prescribed by the DVC-derived BCs, only nodes within the middle $80 \%$ of the scanned specimen were used for comparison. Outliers were

229 removed from the paired QCT-FEM predictions and local DVC results along the $\mathrm{x}, \mathrm{y}$, and $\mathrm{z}$ 230 direction using 5x the Cook's distance (Costa et al., 2017). Slope (m), coefficient of determination $231\left(\mathrm{r}^{2}\right)$ and root-mean-square error (RMSE) were quantified for both loading conditions (compressive and off-axis) and with each BC (idealized-force, idealized-displacement, and DVC-derived). 

and DVC-derived BCs were compared to the experimental applied force. Idealized-force BCs was not included, as the input force required to generate the model was equal to the experimentally measured force. Absolute percentage error was quantified for each specimen and for both loading 237 cases.

\section{$238 \quad 3 . \quad$ Results}

239 The accuracy of predictions generated by the QCT-FEMs was found to be highly sensitive to the 240 boundary conditions simulated. For all four specimens subjected to the compressive load, the 241 performance of QCT-FEMs in predicting experimental local displacements were vastly improved 242 with DVC-derived BCs (Table 2, Figure 5). DVC-derived BCs resulted in the closest match to the 243 experimental results, with ranges of $\mathrm{m}=0.93-1.05, \mathrm{~b}=-0.02-0.01$, and $\mathrm{r}^{2}=0.83-1.00$ for each 244 specimen along the $\mathrm{x}, \mathrm{y}$, and $\mathrm{z}$ direction. Similar agreement between the experimental and QCT245 FEM predictions were not obtained when using idealized BCs. High variations within $\mathrm{m}(-0.13-$ 246 1.66), b (-0.09-0.28) and $r^{2}(0.002-0.93)$ were observed for QCT-FEMs with idealized-force 247 BCs. In addition, high variations in $\mathrm{m}(-0.03-2.84), \mathrm{b}(-0.29-0.75)$, and $\mathrm{r}^{2}(0.001-0.95)$ were 248 observed when using idealized-displacement BCs for all specimens. Overall, RMSE was decreased 249 by two orders of magnitude when using DVC-derived BCs (average RMSE of 4.1 $\pm 0.9 \mu \mathrm{m}, 4.7 \pm 2.2$ $250 \mu \mathrm{m}, 4.8 \pm 2.3 \mu \mathrm{m}$ along $\mathrm{x}, \mathrm{y}$, and $\mathrm{z}$ direction respectively) compared to the idealized-force $\mathrm{BC}$ 251 (average RMSE of $414 \pm 479 \mu \mathrm{m}, 401 \pm 356 \mu \mathrm{m}, 331 \pm 170 \mu \mathrm{m}$ along $\mathrm{x}, \mathrm{y}$, and $\mathrm{z}$ direction 252 respectively) and idealized-displacement BC (average RMSE of $367 \pm 342 \mu \mathrm{m}, 322 \pm 273 \mu \mathrm{m}$, $253175 \pm 96 \mu \mathrm{m}$ along $\mathrm{x}, \mathrm{y}$, and $\mathrm{z}$ direction respectively). 
Table 2: Linear regression results between local displacements predicted by QCT-based FEMs and DVC experimental results due to a compressive load.

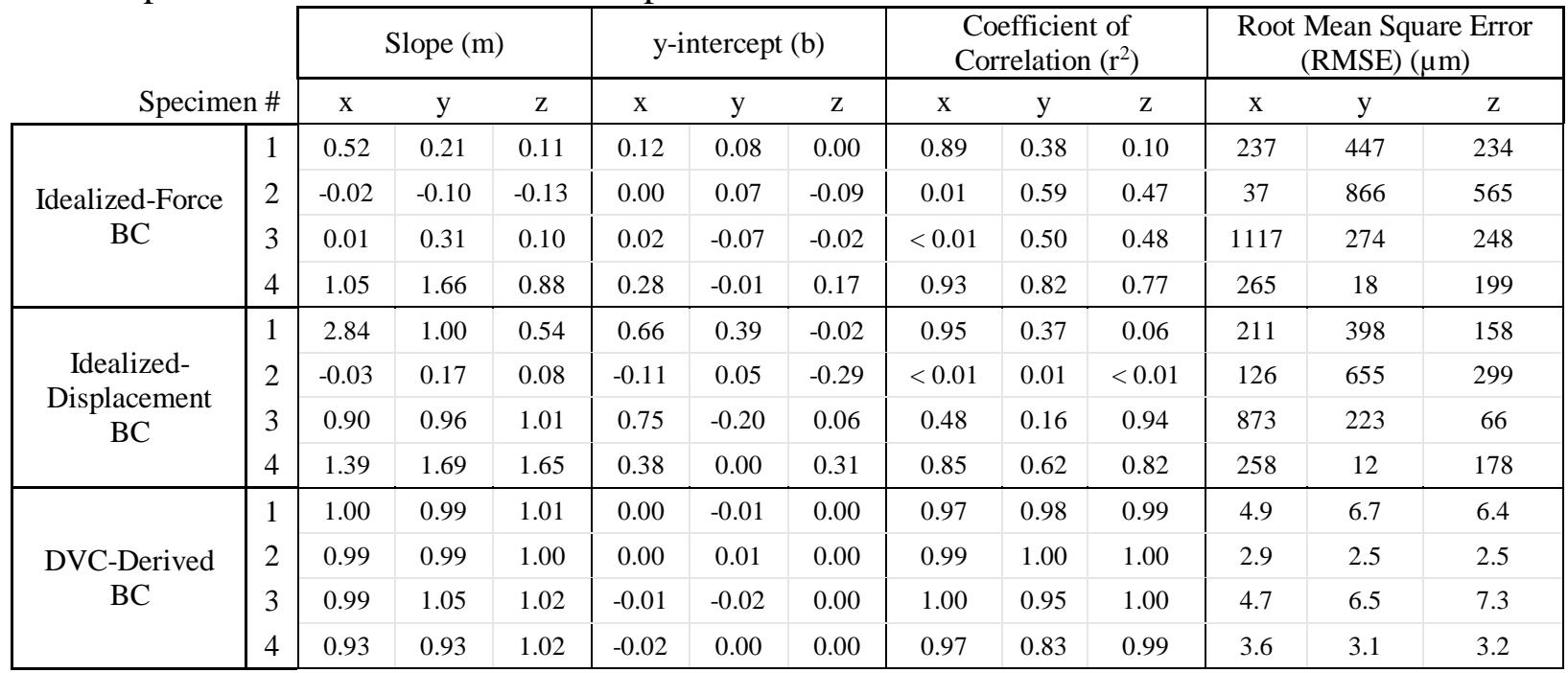

Experimental

Displacements
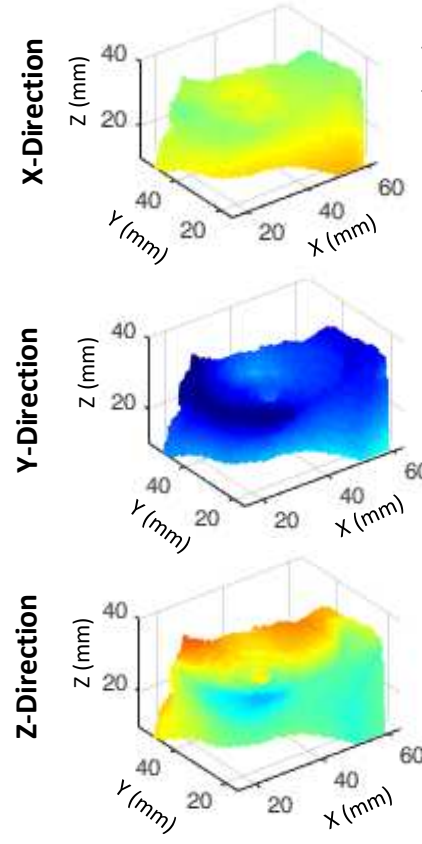

QCT-FEM Predictions

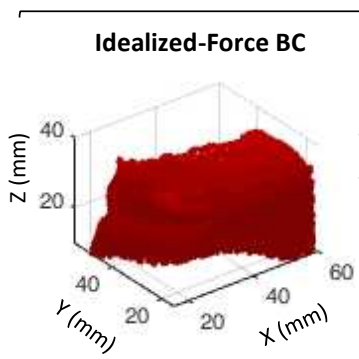

Idealized-Displacement BC

DVC-Derived BC
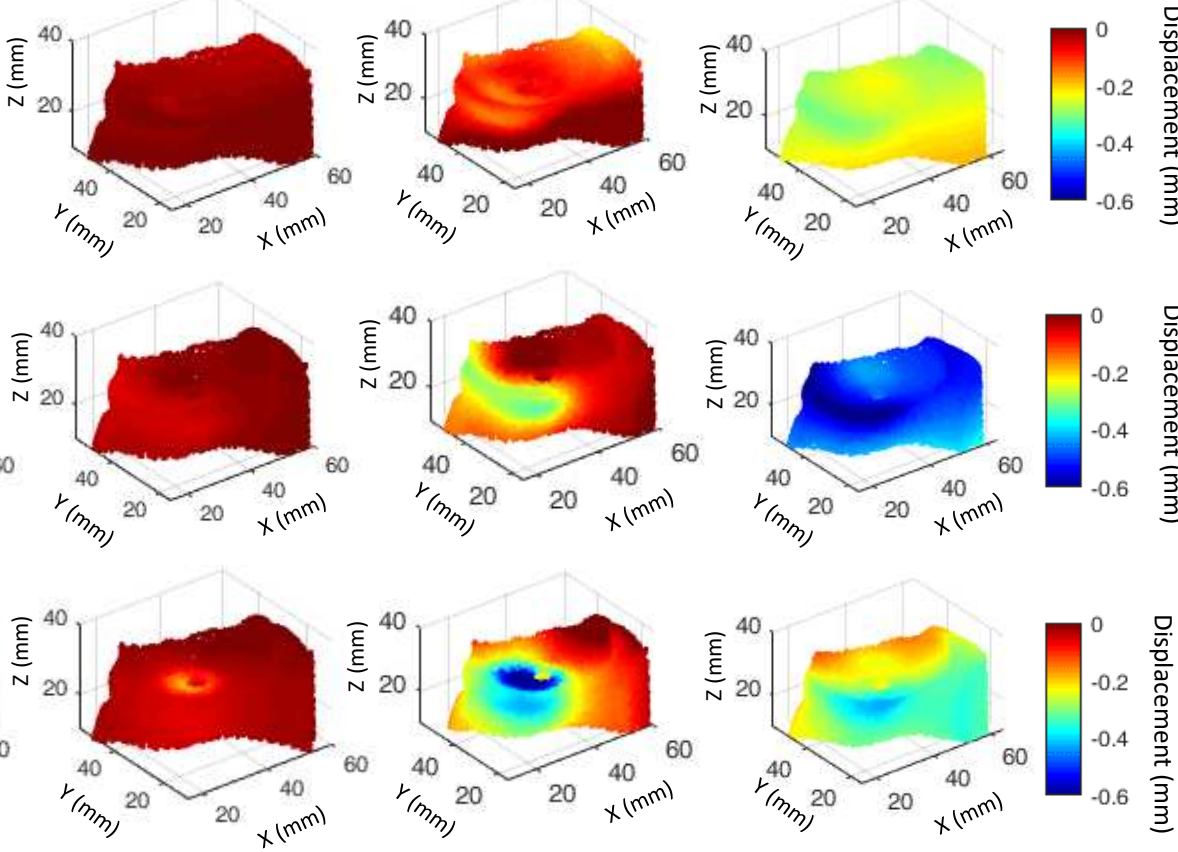

Figure 5: Representative full-field experimental displacements for specimen 1 during a compressive load, compared to predictions generated by a QCT-FEM with idealized-force BC, idealized-displacement BC, and DVC-derived BC. 
For the off-axis load, similar results to the compressive load were observed (Table 3). High

variability in $\mathrm{m}$ (ranges $-0.40-2.35), \mathrm{b}(-0.70-0.98)$, and $\mathrm{r}^{2}(0.002-0.98)$ were observed for both

259 idealized BCs. However, when using DVC-derived BCs, excellent agreement with the experimental results were obtained with ranges of $\mathrm{m}(0.87-1.09), \mathrm{b}(-0.03-0.03)$, and $\mathrm{r}^{2}(0.79-$ measurements was greatly reduced when using DVC-derived BCs (average RMSE of $4.8 \pm 0.7 \mu \mathrm{m}$,

RMSE of $541 \pm 268 \mu \mathrm{m}, 443 \pm 681 \mu \mathrm{m}, 337 \pm 225 \mu \mathrm{m}$ along $\mathrm{x}, \mathrm{y}$, and $\mathrm{z}$ direction) and idealized$\mathrm{z}$ direction).

Table 3: Linear regression results between local displacements predicted by QCT-based FEMs and 268 DVC experimental results due to an off-axis load.

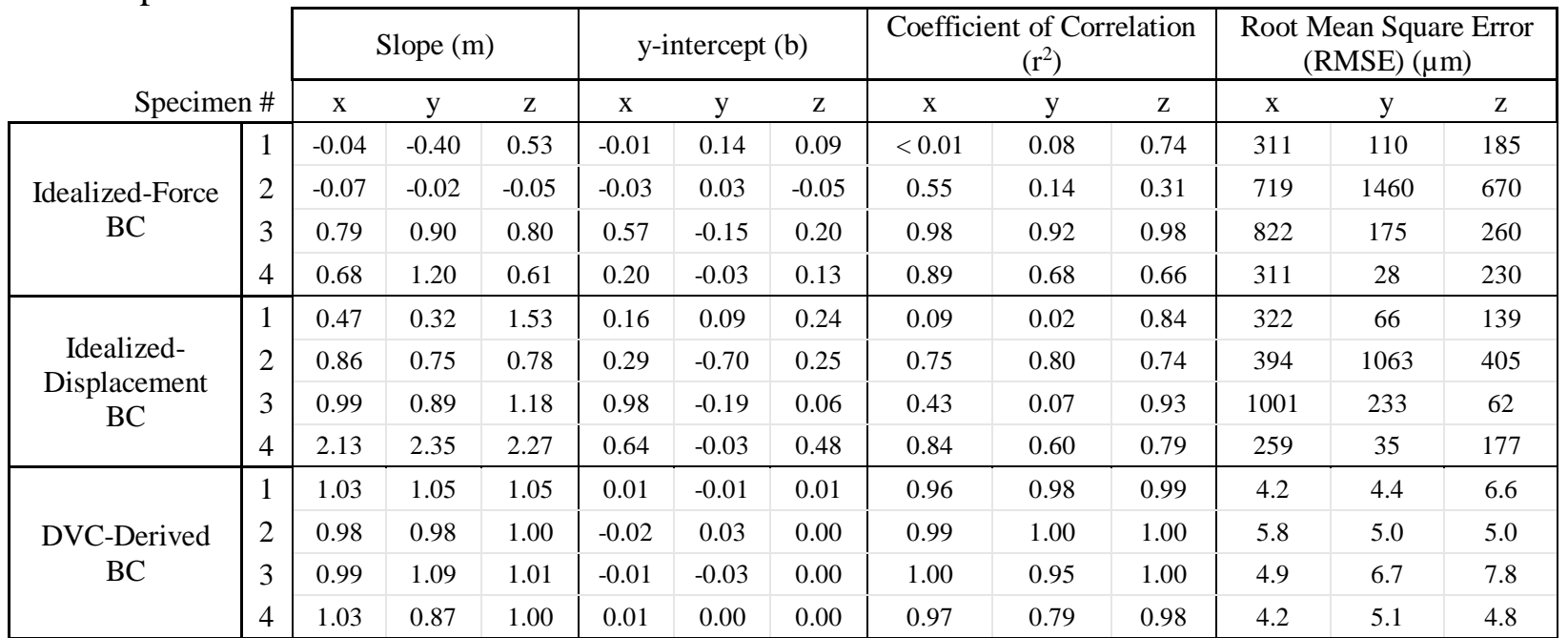

High percentage errors (average error $=333 \%$, range $=169-429 \%$ ) in reaction forces were

271 Comparatively, the percentage error was reduced when using QCT-FEMs with DVC-derived BCs

272 (average error $=32 \%$, range $=8-44 \%$ ). Similar results were observed with an off-axis load, with 
273 higher percentage errors when using idealized-displacement BCs (average error $=350 \%$, range $=$

$274152-520 \%$ ) compared to DVC-derived BCs (average error $=26 \%$, range $=6-50 \%$ ).

\section{4. Discussion}

276 The goal of the current study was to quantify the performance of scapula QCT-FEMs in predicting

277 local displacement measurements obtained from combining digital volume correlation (DVC) and

278 mechanical loading within a microCT scanner. Due to the inability to resolve trabecular

279 microarchitecture at a resolution associated with clinical in-vivo imaging of bone, QCT-FEMs rely

280 on continuum-level assumptions and ignore the geometry of the inner trabecular network.

281 However, the accuracy of local predictions generated by subject-specific vertebra QCT-FEMs has

282 recently been questioned (Hussein et al., 2018; Jackman et al., 2016). The results of the current

283 study found that QCT-FEMs of the scapula can accurately predict local displacement

284 measurements when using DVC-derived BCs. A two orders of magnitude decrease was observed

285 in RMSE, when using QCT-FEMs with simulated DVC-derived BCs compared to the idealized

286 BCs during a compressive or off-axis load. Furthermore, excellent agreement (m ranging from

$287 \quad 0.87-1.09, \mathrm{r}^{2}$ ranging from 0.79 - 1.00) was found between experimental results and QCT-FEM

288 predictions when using DVC-derived BCs, consistent with previous studies performed using $\mu$ -

289 FEMs on different bone structures (Chen et al., 2017; Costa et al., 2017; Oliviero et al., 2018).

290 Reaction forces predicted by the QCT-FEMs with DVC-derived BCs were also within reasonable

291 error (compressive error range 8 - 44\%, off-axis error range 6 - 50\%). The excellent performance

292 of the QCT-FEMs with DVC-derived BCs within the current study may partly be attributed to the

293 fact that loading only within the elastic range was performed, simplifying the QCT-FEMs

294 generated, compared to step-wise fracture loading previously performed (Hussein et al., 2018;

295 Jackman et al., 2016). In addition, inherent differences between the vertebra and scapula, and their 
296 constitutive equations used to assign material properties may contribute to the performance

297 differences in QCT-FEMs of this study compared to previous vertebral studies. Therefore, 298 although excellent agreement between the QCT-FEM predictions and DVC results were observed 299 within the current study in the shoulder, it is unknown whether these findings can be extrapolated 300 to QCT-based FEMs of other joints.

To perform cadaveric experimental loading within a microCT, a secondary objective 302 included the design of a CT-compatible loading device. The robot's ability to generate articular loads within a small working envelope overcame a major design constraint imposed by space 304 restrictions within a CT scanner. Furthermore, controlled loading in 6-dof is a marked 305 improvement over previously developed screw-based CT-compatible devices (Jackman et al., 306 2016; Martelli and Perilli, 2018; Palanca et al., 2016; Sukjamsri et al., 2015). Precision of the DVC 307 measurements was found not to be affected by the hexapod robot, as errors $<2.5 \mu \mathrm{m}$ along each 308 Cartesian direction were recorded during the repeated scans procedure further demonstrating the 309 feasibility of the apparatus.

For QCT-FEMs with idealized-force BCs, local displacement predictions generally 311 underestimated the experimental results. In addition, computed reaction forces required to displace 312 the virtual platen were much higher in QCT-FEMs with idealized-displacement BCs compared to 313 idealized-force BCs. This may suggest that the QCT-FEMs may have been too stiff due to over314 constrained idealized BCs. Only stiffness of the specimen mount (including the PMMA cement) 315 is relevant, since the experimental displacement was measured from microCT rather than from the 316 apparatus itself. Potentially, local DVC displacement measurements could have been applied to 317 the bottom surface of the idealized QCT-FEMs and this may have reduced the observed 318 differences; however, this would require localized displacement measures as an input into the 
QCT-FEMs that may not always be attainable. Nonetheless, stiffness of the experimental setup was not accounted for in this study, as the idealized BCs were modelled according to the literature

321 (Chen et al., 2017; Jackman et al., 2016). Now that it has been observed that QCT-FEMs with

322 DVC-derived BCs can in fact replicate experimental displacement measures, future work could 323 include modelling the experimental apparatus to allow for the QCT-FEMs to become more 324 generalizable. using linear-isotropic material properties subjected to elastic loads. Therefore, further validation is required to investigate failure mechanisms that arise due to loading within the inelastic region. Furthermore, only local displacement measurements and global reaction forces were used to quantify the performance of scapula QCT-FEMs. While strain is a commonly used metric produced by QCT-FEMs to predict failure, experimental strains calculated from microCT-based DVC displacements exhibit higher uncertainties. As the scope of the current study only included evaluating local displacements and global reaction forces, the impact of boundary conditions on other local outcome predictions such as strain was not explored. A Synchrotron light source could

334 be used which may reduce experimental uncertainties (Comini et al., 2019; Palanca et al., 2017);

335 however, this was outside the scope of the current study. Finally, the low sample size $(n=4)$ of 336 this study is a limitation, which was a product of the complex and time-consuming loading and 337 imaging protocol.

The results of the study demonstrate that errors in local displacements predicted by QCT339 FEMs of the shoulder can be minimized using DVC-derived boundary conditions. This work also 340 demonstrated that a novel CT-compatible hexapod robot design was effective for applying 6-dof 341 loading vectors to a scapula while acquiring high-resolution microCT scans in a cone beam 
scanner. Combining volumetric imaging with DVC analysis allowed for the ability to evaluate full-field internal displacement predictions generated by the QCT-FEMs that otherwise could not be captured with traditional surface-based measurement techniques (Dahan et al., 2016; Lin et al., 2016). Further development of these methods should be conducted to examine fracture mechanisms.

\section{Acknowledgements}

The authors would like to thank Aoife Pucchio for her assistance with data collection. This research was supported by a Post Graduate Scholarship from the Natural Sciences and Engineering Research Council of Canada. Jonathan Kusins and Nikolas Knowles are supported in part by a Transdisciplinary Bone \& Joint Training Award from the Collaborative Training Program in Musculoskeletal Health Research at Western University. Enrico Dall'Ara and Melissa Ryan were supported by the Engineering and Physical Sciences Research Council (Grant Number: $\mathrm{EP} / \mathrm{P} 015778 / 1)$.

\section{References}

Allred, J.J., Flores-Hernandez, C., Hoenecke Jr, H.R., D’Lima, D.D., 2016. Posterior augmented glenoid implants require less bone removal and generate lower stresses: a finite element analysis. Journal of shoulder and elbow surgery 25, 823-830. https://doi.org/10.1016/j.jse.2015.10.003

Bay, B.K., Smith, T.S., Fyhrie, D.P., Saad, M., 1999. Digital volume correlation: Three-dimensional strain mapping using X-ray tomography. Exp. Mech. 39, 217-226. https://doi.org/10.1007/BF02323555

Boyin Ding, Stanley, R.M., Cazzolato, B.S., Costi, J.J., 2011. Real-time FPGA control of a hexapod robot for 6-DOF biomechanical testing, in: IECON 2011. Presented at the IECON 2011 - 37th Annual Conference of IEEE Industrial Electronics, Melbourne, AU, pp. 252-257. https://doi.org/10.1109/IECON.2011.6119320

Burkhart, T.A., Andrews, D.M., Dunning, C.E., 2013. Finite element modeling mesh quality, energy balance and validation methods: A review with recommendations associated with the modeling of bone tissue. J Biomech 46, 1477-1488. https://doi.org/10.1016/j.jbiomech.2013.03.022

Chen, Y., Dall'Ara, E., Sales, E., Manda, K., Wallace, R., Pankaj, P., Viceconti, M., 2017. Micro-CT based finite element models of cancellous bone predict accurately displacement once the 
boundary condition is well replicated: A validation study. J Mech Behav Biomed Mater 65, 644651. https://doi.org/10.1016/j.jmbbm.2016.09.014

Comini, F., Palanca, M., Cristofolini, L., Dall'Ara, E., 2019. Uncertainties of synchrotron microCT-based digital volume correlation bone strain measurements under simulated deformation. J Biomech 86, 232-237. https://doi.org/10.1016/j.jbiomech.2019.01.041

Costa, M.C., Tozzi, G., Cristofolini, L., Danesi, V., Viceconti, M., Dall'Ara, E., 2017. Micro Finite Element models of the vertebral body: Validation of local displacement predictions. Plos One 12, 1-18. https://doi.org/10.1371/journal.pone.0180151

Croom, B., Wang, W.-M., Li, J., Li, X., 2016. Unveiling 3D Deformations in Polymer Composites by Coupled Micro X-Ray Computed Tomography and Volumetric Digital Image Correlation. Exp. Mech. 56, 999-1016. https://doi.org/10.1007/s11340-016-0140-7

Croom, B.P., Jin, H., Mills, B., Carroll, J., Long, K., Brown, J., Li, X., 2019. Damage mechanisms in elastomeric foam composites: Multiscale X-ray computed tomography and finite element analyses. Compos Sci Technol 169, 195-202. https://doi.org/10.1016/j.compscitech.2018.11.025

Croom, B.P., Xu, P., Lahoda, E.J., Deck, C.P., Li, X., 2017. Quantifying the three-dimensional damage and stress redistribution mechanisms of braided $\mathrm{SiC} / \mathrm{SiC}$ composites by in situ volumetric digital image correlation. Scr. Mater 130, 238-241. https://doi.org/10.1016/j.scriptamat.2016.12.021

Dahan, G., Trabelsi, N., Safran, O., Yosibash, Z., 2016. Verified and validated finite element analyses of humeri. J Biomech 49, 1094-1102. https://doi.org/10.1016/j.jbiomech.2016.02.036

Dall'Ara, E., Barber, D., Viceconti, M., 2014. About the inevitable compromise between spatial resolution and accuracy of strain measurement for bone tissue: A 3D zero-strain study. J Biomech 47, 2956-2963. https://doi.org/10.1016/j.jbiomech.2014.07.019

Dall'Ara, E., Peña-Fernández, M., Palanca, M., Giorgi, M., Cristofolini, L., Tozzi, G., 2017. Precision of Digital Volume Correlation Approaches for Strain Analysis in Bone Imaged with MicroComputed Tomography at Different Dimensional Levels. Front Mater Sci 4, 1-13. https://doi.org/10.3389/fmats.2017.00031

Du, J., Lee, J.-H., Jang, A.T., Gu, A., Hossaini-Zadeh, M., Prevost, R., Curtis, D.A., Ho, S.P., 2015. Biomechanics and strain mapping in bone as related to immediately-loaded dental implants. J Biomech 48, 3486-3494. https://doi.org/10.1016/j.jbiomech.2015.05.014

Gillard, F., Boardman, R., Mavrogordato, M., Hollis, D., Sinclair, I., Pierron, F., Browne, M., 2014. The application of digital volume correlation (DVC) to study the microstructural behaviour of trabecular bone during compression. J Mech Behav Biomed Mater 29, 480-499. https://doi.org/10.1016/j.jmbbm.2013.09.014

Grassi, L., Isaksson, H., 2015. Extracting accurate strain measurements in bone mechanics: a critical review of current methods. J Mech Behav Biomed Mater 50, 43-54. https://doi.org/10.1016/j.jmbbm.2015.06.006

Gupta, S., Van Der Helm, F.C.T., Sterk, J.C., Van Keulen, F., Kaptein, B.L., 2004. Development and experimental validation of a three-dimensional finite element model of the human scapula. Proc Inst Mech Eng H 218, 127-142. https://doi.org/10.1243/095441104322984022

Hussein, A.I., Louzeiro, D.T., Unnikrishnan, G.U., Morgan, E.F., 2018. Differences in Trabecular Microarchitecture and Simplified Boundary Conditions Limit the Accuracy of Quantitative Computed Tomography-Based Finite Element Models of Vertebral Failure. J Biomech Eng 140, 1-11. https://doi.org/10.1115/1.4038609

Jackman, T.M., DelMonaco, A.M., Morgan, E.F., 2016. Accuracy of finite element analyses of CT scans in predictions of vertebral failure patterns under axial compression and anterior flexion. $\mathrm{J}$ Biomech 49, 267-275. https://doi.org/10.1016/j.jbiomech.2015.12.004

Knowles, N.K., G. Langohr, G.D., Faieghi, M., Nelson, A., Ferreira, L.M., 2019. Development of a validated glenoid trabecular density-modulus relationship. J Mech Behav Biomed Mater 90, 140145. https://doi.org/10.1016/j.jmbbm.2018.10.013 
Lawless, I.M., Ding, B., Cazzolato, B.S., Costi, J.J., 2014. Adaptive velocity-based six degree of freedom load control for real-time unconstrained biomechanical testing. J Biomech 47, 3241-3247. https://doi.org/10.1016/j.jbiomech.2014.06.023

Lin, Z.X., Xu, Z.-H., An, Y.H., Li, X., 2016. In situ observation of fracture behavior of canine cortical bone under bending. Mater. Sci. Eng. C 62, 361-367. https://doi.org/10.1016/j.msec.2016.01.061

Liu, L., Morgan, E.F., 2007. Accuracy and precision of digital volume correlation in quantifying displacements and strains in trabecular bone. J Biomech 40, 3516-3520. https://doi.org/10.1016/j.jbiomech.2007.04.019

Mao, Q., Su, K., Zhou, Y., Hossaini-Zadeh, M., Lewis, G.S., Du, J., 2019. Voxel-based micro-finite element analysis of dental implants in a human cadaveric mandible: Tissue modulus assignment and sensitivity analyses. J Mech Behav Biomed Mater 94, 229-237. https://doi.org/10.1016/j.jmbbm.2019.03.008

Martelli, S., Perilli, E., 2018. Time-elapsed synchrotron-light microstructural imaging of femoral neck fracture. J Mech Behav Biomed Mater 84, 265-272. https://doi.org/10.1016/j.jmbbm.2018.05.016

Nazarian, A., Müller, R., 2004. Time-lapsed microstructural imaging of bone failure behavior. J Biomech 37, 55-65. https://doi.org/10.1016/S0021-9290(03)00254-9

Oliviero, S., Giorgi, M., Dall'Ara, E., 2018. Validation of finite element models of the mouse tibia using digital volume correlation. J Mech Behav Biomed Mater 86, 172-184. https://doi.org/10.1016/j.jmbbm.2018.06.022

Palanca, M., Bodey, A.J., Giorgi, M., Viceconti, M., Lacroix, D., Cristofolini, L., Dall'Ara, E., 2017. Local displacement and strain uncertainties in different bone types by digital volume correlation of synchrotron microtomograms. J Biomech 58, 27-36. https://doi.org/10.1016/j.jbiomech.2017.04.007

Palanca, M., Cristofolini, L., Dall'Ara, E., Curto, M., Innocente, F., Danesi, V., Tozzi, G., 2016. Digital volume correlation can be used to estimate local strains in natural and augmented vertebrae: An organ-level study. J Biomech 49, 3882-3890. https://doi.org/10.1016/j.jbiomech.2016.10.018

Rice, J., Cowin, S., Bowman, J., 1988. On the dependence of the elasticity and strength of cancellous bone on apparent density. J Biomech 21, 155-168. https://doi.org/10.1016/0021-9290(88)900085

Roberts, B.C., Perilli, E., Reynolds, K.J., 2014. Application of the digital volume correlation technique for the measurement of displacement and strain fields in bone: a literature review. Journal of biomechanics 47, 923-934. https://doi.org/10.1016/j.jbiomech.2014.01.001

Schaffler, M.B., Burr, D.B., 1988. Stiffness of compact bone: effects of porosity and density. J Biomech 21, 13-16. https://doi.org/10.1016/0021-9290(88)90186-8

Schneider, C.A., Rasband, W.S., Eliceiri, K.W., 2012. NIH Image to ImageJ: 25 years of image analysis. Nat Methods 9, 671.

Sukjamsri, C., Geraldes, D.M., Gregory, T., Ahmed, F., Hollis, D., Schenk, S., Amis, A., Emery, R., Hansen, U., 2015. Digital volume correlation and micro-CT: An in-vitro technique for measuring full-field interface micromotion around polyethylene implants. J Biomech 48, 3447-3454. https://doi.org/10.1016/j.jbiomech.2015.05.024

Terrier, A., Büchler, P., Farron, A., 2005. Bone-cement interface of the glenoid component: stress analysis for varying cement thickness. Clin Biomech 20, 710-717. https://doi.org/10.1016/j.clinbiomech.2005.03.010

Walker, M.R., Dickey, J.P., 2007. New methodology for multi-dimensional spinal joint testing with a parallel robot. Med Biol Eng Comput 45, 297-304. https://doi.org/10.1007/s11517-006-0158-6

Zauel, R., Yeni, Y.N., Bay, B.K., Dong, X.N., Fyhrie, D.P., 2005. Comparison of the Linear Finite Element Prediction of Deformation and Strain of Human Cancellous Bone to 3D Digital Volume Correlation Measurements. J Biomech Eng 128, 1-6. https://doi.org/10.1115/1.2146001

Zheng, M., Zou, Z., Bartolo, P. jorge D. silva, Peach, C., Ren, L., 2017. Finite element models of the human shoulder complex: a review of their clinical implications and modelling techniques: Finite 
Element Models of Human Shoulder Complex. Int J Numer Method Biomed Eng. 33, e02777.

472

473

474 https://doi.org/10.1002/cnm.2777

475

Zhou, Y., Lamberty, M.A.H., Lewis, G.S., Armstrong, A.D., Du, J., 2018. 3D Full-Field Mechanical Measurement of a Shoulder Bone Under Implant Loading, in: TMS Annual Meeting \&

Exhibition. Springer, pp. 287-293. https://doi.org/10.1007/978-3-319-72526-0_26 